\title{
Theoretical-probabilistic model of the rotary belt grinding process
}

\author{
Sergey Bratan ${ }^{1, *}$, Aleksandr Kolesov ${ }^{1}$, Stanislav Roshchupkin ${ }^{1}$, and Tatyana Stadnik $^{1}$ \\ ${ }^{1}$ Sevastopol State University,33, Universitetskaya St., Sevastopol, 299053, Russia
}

\begin{abstract}
At present, belt grinding is widely used in the leading branches of the national economy. The rotary belt grinding process has a complex stochastic nature, which leads to a dispersion of product quality parameters and does not allow to use all possibilities of this method, due to the lack of an adequate mathematical description of the process. The article is devoted to the development of the theoretical and probabilistic model of the rotary belt grinding process.
\end{abstract}

\section{Introduction}

The transition to market relations in the economy put forward the problems associated with the production of competitive products in engineering, car manufacturing, aircraft manufacturing and other industries. "The consumers need products that meet their needs and expectations" (ISO/CPM 2 9000: 2015). A high level of requirements for modern machines and devices has caused a number of problems associated with the creation of high-performance technological processes that ensure the manufacture of parts with specified quality parameters. The quality parameters determine the functional suitability of the parts and their performance characteristics. Such parameters are formed to the full at the final stages, including grinding and finishing processes. When manufacturing a wide range of parts for machines and devices, it is necessary, on the one hand, to maintain high demands about the quality of their geometric dimensions, roughness and the physical and mechanical state of the surface layer; on the other hand, to increase the productivity of their output according to market requirements. The task is not only to improve the accuracy of manufacturing parts in general, but also to ensure the stability of their parameters. The research in the field of creating technological processes for shaping the working surfaces of precision parts will improve the quality of parts processing, and hence the operational properties of the entire product.

One of the most progressive methods for product processing is grinding with an abrasive belt that moves at a high speed of up to $50 \mathrm{~m} / \mathrm{s}$ [3].

An abrasive belt grinding is widely used in the aircraft-, car- and shipbuilding, in the bearing and woodworking industries, as well as in other branches of the national economy at all stages of the technological process of manufacturing parts: when roughing with the removal of large allowances, when finishing workpieces with low roughness, the required degree of accuracy and quality of the treated surface [4]

The abrasive belt grinding has a number of significant advantages in comparison with the abrasive wheel treatment. Unlike the abrasive wheels with rigid fastening of the cutting grains, the flexible belts make it possible to vary the contact area between the tool and the workpiece, which helps to control the magnitude and character of the contact pressure distribution, the number of cutting elements in the cutting zone in accordance with the physical and mechanical properties of the workpiece and the technical conditions of its processing [5].

In comparison with the abrasive wheel grinding, the belt grinding process is characterized by a higher productivity and a better surface quality of the processed part. The belt grinding provides the roughness $R a 0,63 \div$ 0,08 .

In general, the belt grinding has the following advantages over the abrasive wheel treatment: high grinding performance; greater contact between the belt and the workpiece; a lower contact temperature during grinding; short preparatory-final time. The belt grinding equipment is simple in design and economical in operation. These circumstances make the advantages of abrasive belts undeniable in comparison with the abrasive wheel grinding in finishing stages when processing complex, dimensional and lengthy parts, rolled metal and drawing products $[4,5]$.

The disadvantages of the abrasive belt grinding: less dimensional accuracy and geometric shape during processing; difficulties in processing sharp ridges on parts; relatively low abrasive tape resistance and short service life due to inability to straighten (in most cases); belt extension during operation.

One of the most common methods of belt grinding is grinding with a free strand of the abrasive belt, where the abrasive belt comes into contact with a workpiece on the free strand, resulting in an increase of the contact area of

\footnotetext{
Corresponding author: bratan@sevsu.ru
} 
the belt with the workpiece due to a larger angle of coverage. This method is used for grinding shaped parts, internal and external surfaces, long pipes and slender parts. For processing axisymmetric nonrigid long parts when the rotation of the workpiece is impossible or difficult, the most optimal scheme, from the perspective of achieving surface roughness values $R_{a}=0,63$ and below, is the rotary belt grinding method [3-5].

The processing method (Figure 1) is realized due to a combination of movements of the abrasive belt moving at a speed $V_{l}$, rotation of the faceplate 1 at a speed $V_{g}$, providing a circular feed. The longitudinal feed during one direction grinding is provided by moving the workpiece 5 along its axis, and it is absent from the plunge grinding pattern.

The abrasive tape 6 is located on three rollers - the leading roller 3 , the spreading roller 4 and the driven roller 2 .

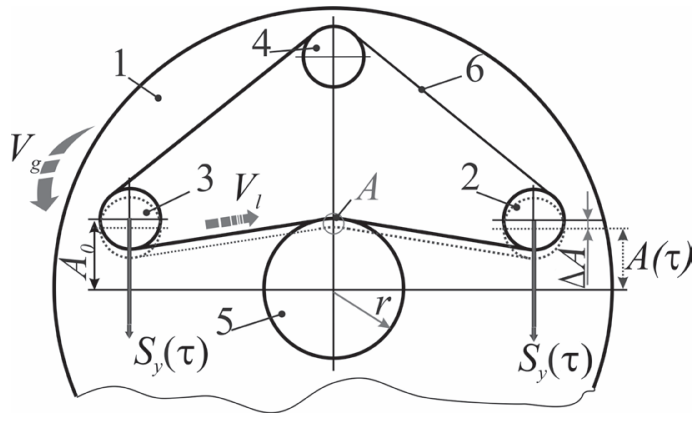

Fig. 1. Pattern of the rotary belt grinding process with two movable rollers. 1 - faceplate; 2 - driven roller; 3 - driving roller; 4 - spreading roller; 5 - workpiece; 6 abrasive belt.

Largely, the rotary grinding makes it possible to provide the required technological parameters of the surfaces to be treated, however, the results are unstable and the shaping process is difficult to control. To stabilize the quality parameters, the technological modes are assigned based on the technologist's experience or the recommendations given in the reference materials. When predicting the state of a technological system (TS), the empirical models of the manufacturing procedure (MP) flow, obtained by the methods of multifactorial experiment planning, are used. The error of such models in some cases reaches $100 \ldots 300 \%$, which does not allow to use them for calculating the cutting modes for rotary belt grinding. At the same time, the process of belt grinding has a complex stochastic nature, which leads to a dispersion of product quality parameters and does not allow to use all possibilities of this method, due to the lack of an adequate mathematical description of the process.

Ensuring the quality and efficiency of the rotary belt grinding operation is possible only on the basis of the development of stochastic models, taking into account the probabilistic nature of the process. A comprehensive solution to these problems is required, and the totality of these problems is an unresolved task to date.
Very often in the simulation, the operation is considered as a dynamic system [6]. One of the possible schemes for the decomposition of an operation as a dynamical system is considered in [7]. According to the functional features, operation is divided into subsystems of the machine, fixtures, tools, workpieces, lubricating and cooling manufacturing agents. Each of the subsystems has its own set of properties, state parameters, development history, the vector of input and output variables, and the vector of perturbing influences.

The central subsystem is the contact area of the workpiece and the tool. The area state parameters include size, shape, chip formation process, material removal rate, wear and abrasive material destruction. The dimensions of the area are in direct proportion with the state of the surface, the dimensions and the spatial arrangement of the abrasive belt and a workpiece. Due to changes in size, spatial location and state of the contacting surfaces over time, the contact area state parameters will change and, as a consequence, the output process parameters [6].

Therefore, the aim of this work is to establish the relationship between the processing modes with the current parameters of the contact area and the output quality parameters for the rotary belt grinding and to develop transient stochastic process models.

\section{Development of the mathematical model of the rotary belt grinding process}

To obtain a mathematical model that allows calculating the output quality parameters, let us consider the interaction process between the abrasive tool and the workpiece at the microlevel in rotary belt grinding (Fig. 2).

The probability of material removal taking into account the initial roughness is determined by the following formula [1]:

$$
P(M)=1-e^{-a(y)-a(y, \tau)},
$$

where $a(y)$ is the indicator determining the material removal probability at level $y$ before the surface comes into contact with the workpiece and the belt; $a(y), \tau$ is the parameter determining the change in the material removal probability at level $y$ in the contact area.

In the grinding process the material is removed in discrete portions, but the number of single sections passing through the cross-section is quite large (from several hundreds to several thousands per section length unit), which allows, when calculating the probability, to move from a discrete model to a continuous one. 


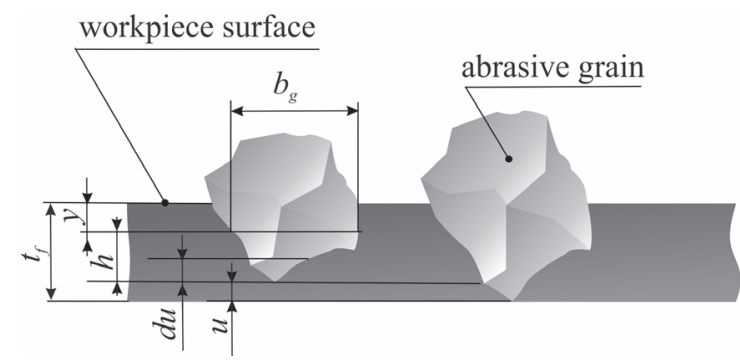

Fig. 2. Interaction pattern of an abrasive tool with a working surface at micro level.

When modeling the grain profile, in the simplest case, the rotation paraboloid determines the width of the vertex profile $b_{g}$ at level $h$ as follows:

$$
b_{g}=2 \sqrt{2 \rho_{g} h} \text {, }
$$

where $\rho_{g}$ is the radius of rounding at the grain top.

The width of the vertex contour corresponding to the given level, in the simulation by a power law, is calculated from the following equation

$$
b_{g}=C_{b} h^{m}=C_{b}\left[t_{f}-y-u\right]^{m},
$$

where $C_{b}$ and $m$ are the coefficient and the exponent (determined experimentally).

With time $\Delta \tau$ a section is rotated at the angle $\Delta \varphi$ and through it a section with the arc length of $V_{k} \Delta \tau$ passes. Out of the total number of grains having passed through the section, the profile width $b_{g}$ will have the grains whose vertices are located in the layer $1 \Delta u V_{k} \Delta \tau$ of the belt. The number of such vertices is calculated according to their distribution density along the depth of the tool $f(u)$.

The number of grains having passed through section $y$ within the time $\Delta \tau$ :

$$
\Delta \lambda=V_{k} n_{g} f(u) \Delta u \Delta \tau,
$$

where $n_{g}$ is the number of grains per unit volume of the tool working layer; $V_{k}$ is the circumferential speed of the tool; $f(u)$ is the distribution of the tool working grains in depth, which can be approximated by various dependencies, for example, by the power law [6]:

$$
f(u)=\frac{(\chi+m-0.5)}{H_{u}^{\chi+m-0.5}} u^{\chi-1},
$$

where $\chi$ and $m$ are parameters of the rotation paraboloid.

The change in the parameter $a(y, \tau)$ is determined by the increment in the sum of the transverse dimensions of the abrasive grain profiles:

$$
\Delta a(y, \tau)=K_{c} n_{g} b_{g} f(u) \Delta u \Delta \tau,
$$

where $K_{c}$ is the chip formation coefficient, taking into account the fact that not all the material is removed from inside a machining mark, and some of it is displaced and forms marks along the edges.

We obtain the integral equation for calculating the parameter $a(y, t)$ in the contact area

$$
a(y, \tau)=V_{k} K_{\mathrm{c}} n_{\mathrm{g}} \int_{t_{0}}^{t_{f}} \int_{0}^{-y} b_{g} f(u) d u d \tau
$$

After integration with respect to $u$ and $\tau$ of dependence (1), we obtain:

$$
a(t)=\frac{0,78 \pi K_{c} \sqrt{2 \rho_{g}} V_{k} n_{g} S_{y}{ }^{2}\left(t-t_{0}\right)^{3}}{2 H_{u}^{1,5}} .
$$

Given that for the outer surface of the part $(y=0)$, $P(\bar{M})=\beta_{0}$ when multiplying $\left.P_{1.2}(\bar{M})=P_{I}(\bar{M}) \cdot P_{2}(\bar{M})\right)$, we obtain the dependences for the probabilities of the material removal failure for the plunge grinding pattern:

$$
1-\beta_{0}=\exp \left\{-a_{0}-\frac{0,78 \pi K_{c} \sqrt{2 \rho_{g}} V_{k} n_{g} S_{y}^{2}\left(t-t_{0}\right)^{3}}{2 H_{u}^{1,5}}\right\}
$$

Further, after taking the logarithm of the equation (2), we obtain:

$$
\ln \frac{1}{1-\beta_{0}}+a_{0}=-\frac{0,78 \pi \mathrm{K}_{\mathrm{c}} \sqrt{2 \rho_{\mathrm{g}}} \mathrm{V}_{\mathrm{k}} \mathrm{n}_{\mathrm{g}} S_{y}^{2}\left(t-t_{0}\right)^{3}}{2 \mathrm{H}_{\mathrm{u}}^{1,5}}
$$

The obtained formula (3) allows to predict not only the radial metal removal, but also the output quality parameters for the rotary belt grinding.

While going through the contact area surface of the part and the belt, the levels will shift to the center of the part. In this case, the material removal value is numerically equal to the level shift with the removal probability $P(V)=\beta_{M}$, and is calculated according to the following formula:

$$
P(M)=1-\exp \left(-\frac{\Theta_{M}}{S_{y}}\left[S_{y}\left(t-t_{0}\right)\right]\right)^{3}=1-P(\bar{M})
$$

where $\Theta_{M}=\frac{0,78 \pi K_{c} \sqrt{2 \rho_{g}} V_{k} n_{g}}{2 H_{u}^{1,5}}$.

When the grains continuously come into contact with the workpiece material, for particular values $\beta_{M}=0,9973$, and $t_{f}=u$ (4) can be rewritten as follows: 


$$
\ln 0,0027=-\frac{\Theta_{M} t_{f}{ }^{k}}{S_{y}} .
$$

Based on formula (5), we obtain an equation that allows one to calculate the value of the layer in which the surface roughness is distributed:

$$
H=\left[-\frac{\ln 0,0027 S_{y}}{\Theta_{M}}\right]^{\frac{1}{k}} .
$$

The value of the layer in which the surface roughness is distributed in rotary belt grinding is numerically equal to the actual infeed depth and is directly proportional to the tool feed.

For a steady grinding process where a particular value of the coefficient $k=3$, the value of the layer in which the roughness is distributed is calculated according to the function:

$$
H=\left[\frac{47,31 S_{y} H_{u}{ }^{1.5}}{\pi K_{C} n_{g} V_{k} \sqrt{2 \rho_{g}}}\right]^{\frac{1}{3}} .
$$

The temperature deformation value can be taken into account by the coefficient.

Function (6) makes it possible to obtain an equation for the material removal calculation

$$
Q_{\Sigma}=t_{f}-\left[\frac{47,31 S_{y} H_{u}^{1.5}}{\pi K_{C} n_{g} V_{k} \sqrt{2 \rho_{g}}}\right]^{\frac{1}{3}} .
$$

Assuming that the profile of the ground surface can be a stationary random process with a normal ordinate distribution, we obtain an equation for estimating the values of the roughness altitude parameters.

The average arithmetic deviation of the profile $R_{a}$ can be calculated (see Figure 3 ) taking into consideration the area of the protrusions $F_{1}$ and the area of the depressions $F_{2}$ measured from the profile midline.

As

$$
d F_{1}=t_{p} l d y_{m} \text { and } d F_{2}=\left(1-t_{p}\right) l d y_{m}
$$

so

$$
R_{a}=\int_{o}^{\infty} t_{p} d y_{m}+\int_{\infty}^{o}\left(1-t_{p}\right) d y_{m}
$$

where $l$ is the length of the base section, mm; $y_{m}$ is the distance from the points of the profile to its midline.

Calculation of $R_{a}$ according to this function is possible when using numerical methods of integration.

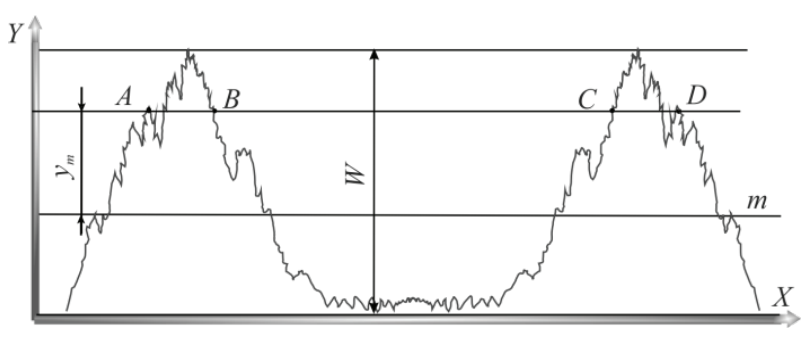

Fig. 3. Profilogram for calculating the distribution function of the profile ordinates of the treated surface.

The function for calculating $R_{a}$ is simplified if we use the law of stationarity and normality of the random process, considered in this work, describing the profile ordinates.

For such a process, the mathematical expectation of the mean arithmetic deviation of the profile is proportional to the mean square deviation $\sigma_{y_{m}}$ of the random ordinates $y_{m}$ of the profile points [1].

$$
M\left(R_{a}\right)=\sqrt{\frac{2}{\pi} K(0)}=\sqrt{\frac{2}{\pi} \sigma_{y m}},
$$

where $K(0)$ is the value of the correlation function for an interval $x_{2}-x_{1}=0$ is equal to the variance of the random variable $y_{m}$.

To determine the root-mean-square deviation $\sigma_{y m}$, let us calculate the function $F_{Y_{m}}\left(Y_{m}\right)$ and the probability density $f_{Y_{m}}\left(Y_{m}\right)$ of distances $Y_{m}$ from the midline to the points of the profile ground surface.

$$
\begin{gathered}
F_{y m}\left(y_{m}\right)=P\left(y<y_{m}\right)=\lim _{l \rightarrow \infty} \frac{l-\sum b_{m i}}{l}=P(M), \\
f\left(y_{m}\right)=\frac{3 \Theta_{M}}{S_{y}}\left(W_{m}+y_{m}\right)^{2}\left\{\exp \left[-\frac{\Theta_{M}\left(W_{m}+y_{m}\right)^{3}}{S_{y}}\right]\right\}
\end{gathered}
$$

where $W_{m}$ is the distance from the deepest depression to the profile midline.

For a normal process where $Y_{m}=0$, $P(M)=0,5[88]$ :

$$
f\left(y_{m}\right)=\frac{1}{\sigma_{y} \sqrt{2 \pi}} .
$$

On basis of this, the second part of the equation (8), separated by curly braces, is equal to 0.5 , and the average arithmetic deviation of the profile, taking into account the equation (7), is defined as:

$$
R_{a}=\frac{2 S_{y}}{\pi \Theta_{M} 3 W_{m}{ }^{2}} .
$$


Thus, when determining $R_{a}$ one must first find the value $W_{m}$. In the general case, it is defined as the solution to the equation:

$$
\Theta_{M} \sum_{i=0}^{n}\left(W_{m}-\sum_{i=1}^{i} \Delta Q_{i}\right)^{2.5}=\ln 2
$$

The solution of the equation (9) is possible only on the basis of numerical methods application, and this greatly complicates the calculation of the surface roughness parameters.

Let us note that formula (7) has a simple analytical form and only the calculation of $W_{m}$ requires the use of numerical methods.

In [6], the function for approximate calculations of the profile arithmetic mean deviation is recommended:

$$
W_{m}=\frac{H}{2} .
$$

Then the function for $R_{a}$ calculation takes the form of

$$
R_{a}=\frac{2 S_{y}}{3 \pi \Theta_{M}(H / 2)^{2}}
$$

After plugging (6) into (11) we obtain:

$$
R_{a}=\frac{S_{y}^{\frac{1}{3}}}{6 \pi \Theta_{M} Z^{2}}
$$

where $Z=\left[\frac{28,895 H_{u}^{1.5}}{\pi K_{C} n_{g} V_{k} \sqrt{2 \rho_{g}}}\right]^{\frac{1}{3}}$.

Equation (12) reflects the physical nature of the surface formation process and corresponds to the basic principles of the dimensional theory.

The complex parameter that combines the altitude and step parameters of the roughness is the relative reference length of the profile. By definition, this parameter coincides with the probability of metal removal failure. When $t_{f}=$ const the formula changes as follows:

$$
t_{p j}=\exp \left[-\frac{A K_{C} n_{g} V_{k} \sqrt{2 \rho_{g}} S_{y}^{2} t^{3}}{8 H_{u}^{1.5}}\right] .
$$

In addition to roughness, the depth of the defective layer is related to the surface quality parameters:

$$
h_{d}=\alpha \xi\left(q-q_{O}\right)\left(\frac{h_{q}}{V_{l}}\right)^{\beta}
$$

where $q_{0}$ is the defect-free level of the heat flux density; $q$ is the heat flow; $h_{q}$ is the half-width of the heat source; $\beta$ is the coefficient determined by the nature of heat release $(\beta=0.8, \ldots, 0.9) ; \alpha$ is the coefficient, depending on the thermal properties of the belt and the workpiece; $\xi$ is the coefficient that takes into account the influence of the electric field on the depth of the defective layer.

The half-width of the heat source

$$
h_{q}=0,5 \frac{\pi \cdot R_{w} \cdot \varepsilon}{180},
$$

where $R_{w}$ is the workpiece radius; $\varepsilon$ is the coverage angle of the workpiece with the abrasive belt.

The coverage angle of the workpiece with the abrasive belt (Figure 4):

$$
\varepsilon=2 \cdot \arccos \left[\frac{\left(R_{1}+R_{n}\right) x_{1}+y_{1} \cdot \sqrt{y_{1}^{2}+x_{1}^{2}-\left(R_{1}+R_{w}\right)^{2}}}{y_{1}^{2}+x_{1}^{2}}\right] .
$$

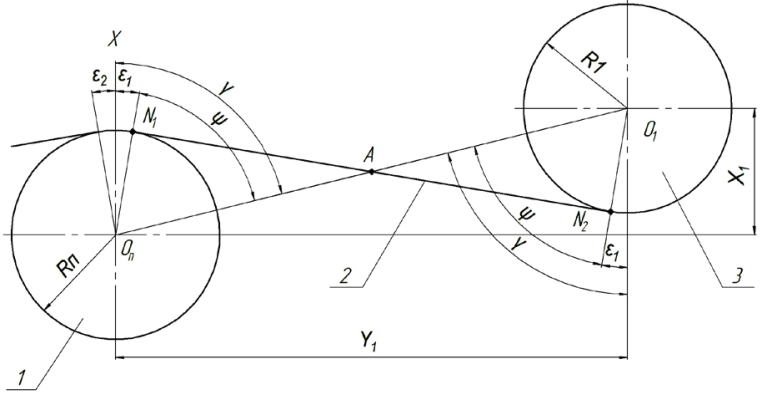

Fig. 4. Scheme for calculation of the coverage angle of the workpiece with the abrasive belt.

Plugging (14) into (13) we obtain a function for calculating the depth of the defect layer:

$$
h_{d}=\alpha \xi\left(q-q_{O}\right)\left(\frac{0,5 \pi \cdot R_{w} \cdot(\varepsilon / 180)}{V_{l}}\right)^{\beta} .
$$

\section{Conclusions}

A stochastic model of the rotary belt grinding process is developed. It takes into account the probabilistic nature of the process and allows to calculate the output quality parameters bearing in mind the changes in the system properties over time.

\section{References}

1. Yu. Novoselov, Dynamics of surface formation in abrasive processing (Publishing House: LAP LAMBERT AcademicPublishing, 2017).

2. S. Korchak, Productivity of the grinding process of steel parts (Car Manufacturing, Moscow, 1974)

3. S. Shilyaev, A. Ivanov, Analysis of constructive and technological parameters of the rotary belt grinding device on basis of studies of the relative movement 
dynamics of an abrasive tape, Science and Education: scientific edition of the Bauman MSTU, 6 ( 2011)

4. A. Koryazhkin, D. Volkov, Stochastic model of the abrasive belt wear for grinding the compressor blades of the gas turbine engine, Vestnik RGATA, 2 (2011)

5. K. Chubukov, S. Shilyaev, Development of a forming pattern for rotary belt grinding, Topical issues of machine tool construction: materials of the All-Russian Scientific and Practical Conference. Izhevsk: Innova (2013)

6. S. Bratan, Technological fundamentals of quality maintenance and stability improvement of highperformance finishing and fine grinding, Thesis of Dr. of Sciences (Odessa, 2006)

7. S. Bratan, Quality maintenance and stability improvement of finishing and fine grinding, Cutting and tools in technological systems. Int. Scient.-Tech. Collection, 68. (2005)

8. M. Jackson, Machining with Abrasives (Springer, New York, 2011) 